\title{
Convergent structure and function of mycelial galleries in two unrelated Neotropical plant-ants
}

\author{
V. E. Mayer ${ }^{1} \cdot$ J. Lauth ${ }^{2} \cdot$ J. Orivel ${ }^{2}$
}

Received: 29 September 2016 / Revised: 10 February 2017 / Accepted: 22 February 2017 / Published online: 11 March 2017

(C) The Author(s) 2017. This article is an open access publication

\begin{abstract}
The construction process and use of galleries by Azteca brevis (Myrmicinae: Dolichoderinae) inhabiting Tetrathylacium macrophyllum (Salicaceae) were compared with Allomerus decemarticulatus (Myrmicinae: Solenopsidini) galleries on Hirtella physophora (Chrysobalanaceae). Though the two ant species are phylogenetically distant, the gallery structure seems to be surprisingly similar and structurally convergent: both are pierced with numerous holes and both ant species use Chaetothyrialean fungi to strengthen the gallery walls. Al. decemarticulatus is known to use the galleries for prey capture and whether this is also the case for Az. brevis was tested in field experiments. We placed Atta workers as potential prey/threat on the galleries and recorded the behaviour of both ant species. We found considerable behavioural differences between them: Al. decemarticulatus was quicker and more efficient at capture than was $A z$. brevis. While most Atta workers were captured after the first $5 \mathrm{~min}$ by Al. decemarticulatus, significantly fewer were captured by Az. brevis even after 20 min. Moreover, the captured Atta were sometimes simply discarded and not taken to the nest by Az. brevis. As a consequence, the major function of the galleries built by
\end{abstract}

Electronic supplementary material The online version of this article (doi:10.1007/s00040-017-0554-y) contains supplementary material, which is available to authorized users.

\section{E. Mayer}

veronika.mayer@univie.ac.at

1 Department of Botany and Biodiversity Research, Division of Structural and Functional Botany, University of Vienna, Rennweg 14, 1030 Vienna, Austria

2 CNRS, UMR Ecologie des Forêts de Guyane, AgroParisTech, CIRAD, INRA, Université de Guyane, Université des Antilles, Campus Agronomique, BP316, 97379 Kourou Cedex, France
Az. brevis may, therefore, be defense against intruders in contrast to Al. decemarticulatus which uses them mainly for prey capture. This may be due to a higher need for protein in Al. decemarticulatus compared to coccid-raising Az. brevis.

Keywords Ant-plant interaction - Ambush behaviour - Allomerus decemarticulatus - Azteca brevis . Chaetothyriales $\cdot$ Hirtella physophora $\cdot$ Runway galleries . Tetrathylacium macrophyllum

\section{Introduction}

The construction of independent nests, galleries, shelters, and inner walls that divide nests into separate cavities is a common feature in ants, particularly in arboreal species, built to provide colonies and their resources protection from climatic stress, parasites, and predators (Anderson and McShea 2001). Most species use carton made from plant fibres and debris or soil particles as building material. In the Neotropics, species from the genus Allomerus (Formicidae: Myrmicinae), as well as a species from the genus Azteca (Formicidae: Dolichoderinae), Az. brevis Forel, are known to build particular galleries on their hostplant stems; these galleries are pierced with holes (Dejean et al. 2004, 2005; Mayer and Voglmayr 2009). Moreover, the galleries of these ant species are reinforced with a dense net of fungal hyphae, which have been identified as members of the ascomycete order Chaetothyriales (Mayer and Voglmayr 2009; Nepel et al. 2014; Ruiz-González et al. 2011). Such apparent similarities in the galleries built by ant species from phylogenetically distant genera belonging to different subfamilies raise the question whether the construction process and the function of the holes are also 
similar. For Allomerus spp., it is known that the galleries are used for prey capture with ant workers waiting inside the galleries with their heads just under the holes and mandibles wide open. Prey that walk over or settle on the gallery are grasped by their legs, antennae, or wings, stretched against the gallery, killed, and taken to the nest (Dejean et al. 2005).

However, it has never before been investigated how $A z$. brevis uses its galleries, and whether these galleries have a function in prey capture analogous to those built by Allomerus. Therefore, we investigated the similarities and differences in the behaviour of the two ant species. We compared (1) the construction process and (2) experimentally tested whether the holes in the galleries built by Az. brevis have a prey capture function analogous to those built by $A l$. decemarticulatus.

\section{Materials and methods}

\section{Species and study sites}

Azteca brevis (Forel 1899) (Dolichoderinae) was studied in the Parque Nacional Piedras Blancas (Costa Rica) on Tetrathylacium macrophyllum growing near the La Gamba Biological Research Station $\left(08^{\circ} 42^{\prime} 46^{\prime \prime} \mathrm{N}, 83^{\circ} 12^{\prime} 90^{\prime \prime} \mathrm{W}\right)$ $(n=4)$, along Rio Sardinal $\left(08^{\circ} 43^{\prime} 48^{\prime \prime} \mathrm{N} 8^{\circ} 13^{\prime} 28^{\prime \prime} \mathrm{W}\right.$; $n=3)$ and along Rio Bolsa $\left(08^{\circ} 41^{\prime} 06^{\prime \prime} \mathrm{N} 83^{\circ} 10^{\prime} 35^{\prime \prime} \mathrm{W}\right.$; $n=2$ ) in a lowland primary rainforest characterised by high annual rainfall $(>5000 \mathrm{~mm})$. Az. brevis is a red-brown ant, ca. 4-mm-long, known only from the Pacific side of Costa Rica, mostly in the wet forests of the southern Pacific lowlands (Longino 2007a). The colonies are polydomous with cauline nests in the live stems of trees (Supplementary Fig. S1). All of the nesting chambers inside the stems are connected externally by an extensive system of galleries made of a characteristically black carton with small, circular holes. Az. brevis attends large populations of coccoid Hemiptera of the genus Cryptostigma (P. Gullan, pers. com.) inside the stems and in the runway tunnels (Supplementary Fig. S1C). Colonies have been found on T. macrophyllum (Salicaceae), a tree with an average height of about $8 \mathrm{~m}$ (but reaching a maximum height of 15-20 m) that grows on the Pacific slopes of Central and South America from southern Costa Rica to Ecuador with its centre of distribution in the Colombian Chocó (Supplementary Fig. S1A). It grows preferentially on steep slopes near rivers and streams in the primary forest (Janzen 1983). The domatia are located on the stems and develop from the partial degeneration of the pith. In the study area, the plant can be colonised by various ant species (Tennant 1989), but $A z$. brevis is the only one which actively enlarges the domatia by excavating the remaining pith (Schmidt 2001).
Allomerus decemarticulatus was studied in the area of Petit Saut, Sinnamary, French Guiana $\left(05^{\circ} 03^{\prime} 30^{\prime \prime}\right.$ $\left.\mathrm{N}, 52^{\circ} 58^{\prime} 34^{\prime \prime} \mathrm{W}\right)$ in a lowland primary rainforest. $A l$. decemarticulatus (Mayr 1878) (Myrmicinae) are minute $(1.5-1.8 \mathrm{~mm})$, tawny-coloured ants that live strictly on antplants. In the study area, Al. decemarticulatus is the main obligatory inhabitant of Hirtella physophora (Chrysobalanaceae) with a single mature colony per plant; this ant species has never been found associated with other myrmecophytic species (Grangier et al. 2009). H. physophora is a long-lived understory treelet that has leaves with a pair of domatia at the base of each lamina (Leroy et al. 2008; Orivel et al. 2011). The species occurs mostly in patches on the upper slopes of hillsides (Solano et al. 2003).

\section{Investigation of the galleries' structure and construction process}

Inner gallery width, height, and hole diameter were measured with calipers. The measurements were taken from 2-cm-long pieces of carton cut from the galleries of four different colonies of $A z$. brevis and two colonies of $A l$. decemarticulatus. Head width, head length (including the mandibles), and the total body length of Al. decemarticulatus $(n=10)$ and Az. brevis $(n=5)$ workers were also measured.

The construction process of the carton galleries was studied for 15 different $A z$. brevis colonies during both the dry and the rainy seasons. We removed $10-\mathrm{cm}$-long sections of the carton galleries and recorded the reconstruction of the galleries by taking a photograph twice daily during 2 weeks. If recently developed branches of the host tree $T$. macrophyllum were easily accessible, we also monitored the continuation of the galleries on these new shoots.

Similar observations were made on ten Al. decemarticulatus colonies during the rainy season. The Al. decemarticulatus galleries were removed from the plant and gallery reconstruction was observed during 2 weeks. We documented the construction by photographing the galleries every day.

\section{Testing the function of the galleries of $\mathrm{Az}$. brevis and $\mathrm{Al}$. decemarticulatus}

To test the role of the Az. brevis galleries and compare it with that of $A l$. decemarticulatus galleries, leaf-cutter ants (e.g. Atta colombica or At. cephalotes) were taken from nearby nests and placed on the galleries to examine the ants' behaviour towards potential invaders or prey. Atta individuals are among the size range of prey that $A l$. decemarticulatus can capture (Dejean et al. 2005). Since Az. brevis workers are even larger than Al. decemarticulatus 
workers, we hypothesised that they should also be able to capture Atta ants.

The experiment was conducted on ten $A l$. decemarticulatus/H. physophora and nine Az. brevis/T. macrophyllum associations. For each plant, three separate and easily accessible branches were selected. One Atta individual was carefully placed with tweezers on an Allomerus-occupied $H$. physophora branch as well as on the upper side of an Azteca-occupied T. macrophyllum branch. The fate of the Atta ants was recorded after four time intervals $(5,10,20,30 \mathrm{~min})$ and classified into one of three different categories: (1) remained free, (2) captured by the inhabiting ants, or (3) escaped from the tree by either dropping or running away. In a control experiment, the carton galleries of three branches from the same trees as before were destroyed and the behaviour of Az. brevis towards the leaf-cutter ants monitored. Az. brevis did not attack Atta individuals that were not on the carton galleries.

Through a Mann-Whitney $U$ test for independent groups with non-parametric data, we tested the null hypothesis that the capture efficiency of Al. decemarticulatus and $\mathrm{Az}$. brevis is the same for each time interval. The test was performed with IBM SPSS Statistics 23.

\section{Results}

\section{Gallery structure, construction material, and process}

For both species, the galleries were similar; i.e., an arched tunnel 2-mm-high and with an inner width of 3-4 mm. For Azteca-as well as Allomerus-built galleries, wall thickness was $0.2 \mathrm{~mm}$, and the walls were perforated with \pm evenly distributed circular holes which were ca. 1-mm-wide for both species, and separated by a distance of $1.3-6.0 \mathrm{~mm}$ for Az. brevis galleries and 2.0-4.5 $\mathrm{mm}$ for Al. decemarticulatus galleries (Fig. 1d, h; supplementary Table 1). The diameter of the holes was slightly larger than the workers' heads with on average $0.96 \mathrm{~mm}$ for $A z$. brevis galleries and $0.55 \mathrm{~mm}$ for Al. decemarticulatus galleries (supplementary
Fig. 1 Construction of the carton galleries by Az. brevis (a-d) and Al. decemarticulatus (e-h). Both ant species start by clearing a path; $A z$. brevis also scrapes tissue from the bark (arrows) (a, e). Az. brevis uses chewed plant tissue to build rows of parallel pillars (b) and fills the space between (c). Holes are left during the construction process (d). Al. decemarticulatus clears a path by cutting the trichomes (e), which are then used to form the vault of the galleries $(\mathbf{f}-\mathbf{g})$. As for Az. brevis, the holes are not pierced after construction but left open during the process (h). The construction process was observed in the field in Costa Rica (Az. brevis) and in French Guiana (Al. decemarticulatus)
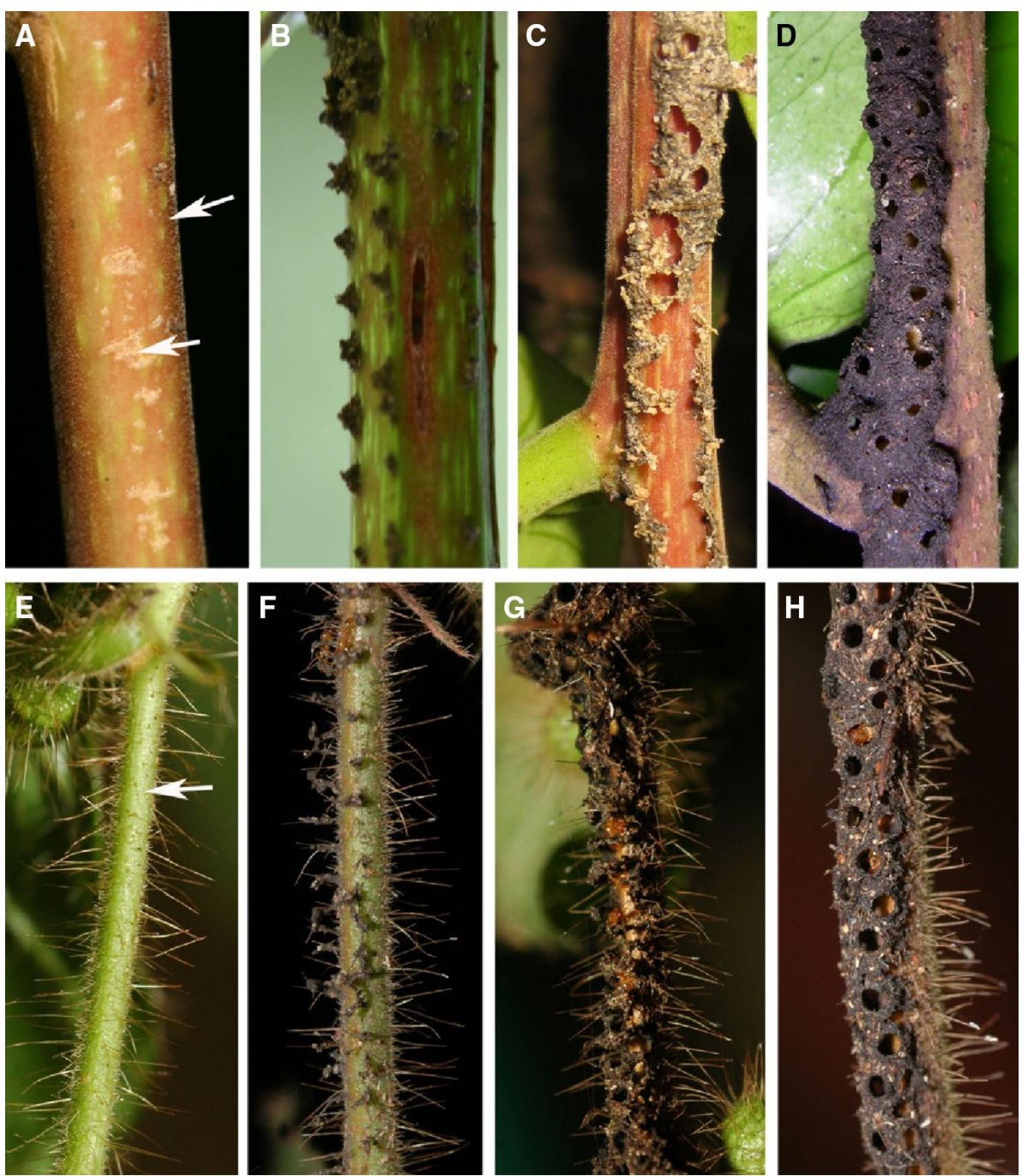
Table 1), allowing the ants to enter and exit the galleries. For Az. brevis, the holes were lined with a bulky ring (Figs. 1d, 2a, b). The galleries usually covered the underside of the branches (Fig. S1, supplementary material), including the entrances to the domatia. The holes were not pierced into completed galleries, but were made during the galleries' construction (Fig. 1d, h).

For both ant species, new galleries were built almost exclusively with plant material. However, the material itself and the way they used it differed considerably. Az. brevis formed pellets from pieces of bark or epiphylls taken from the surface of their host-plant's branches as well as pithy tissue obtained from excavating the branches (Fig. 1a) with which they first built two parallel rows of pillars (Fig. 1b), then a wall between each pair of adjacent pillars, and finally an arch to form the vault of the galleries (Fig. 1c). During the dry season, the construction progress was slow and even stopped after several days without rain, whereas, during the rainy season, up to $4 \mathrm{~cm}$ of gallery were built in a day.

Allomerus decemarticulatus workers did not use bark, epiphylls, or pithy tissue. Instead, they cut trichomes from the surface of the internodes located between the leaf pouches (Fig. 1e) and assembled them together to form the vault of the gallery. Adjacent, uncut trichomes served as pillars, and pellets made from tissue scratched off the inner domatia walls were pasted along the trichomes in the gallery (Fig. 1e-g) (Dejean et al. 2005; Ruiz-González et al. 2011). Then, the ants pasted pellets of hyphae from older gallery sections onto the plant material on newly built parts. The fungus then colonised the galleries, reinforcing them.

\section{Function of the galleries}

When the branches with carton galleries were disturbed, workers of the respective Az. brevis colony adopted positions beneath the holes with their mandibles wide open (Fig. 2a). After the first $5 \mathrm{~min}$ of the experiment, wherein Atta workers were placed on branches with galleries, $77.8 \%$ of the individuals were still free, only $18.5 \%$ were immediately captured, and $3.7 \%$ escaped by simply dropping off the tree. The capture rate increased constantly: $37.0 \%$ were captured and immobilised after $10 \mathrm{~min} ; 63.0 \%$ after $30 \mathrm{~min}$ (Figs. 2c, 3). After $30 \mathrm{~min}, 3.7 \%$ were still free and $33.0 \%$
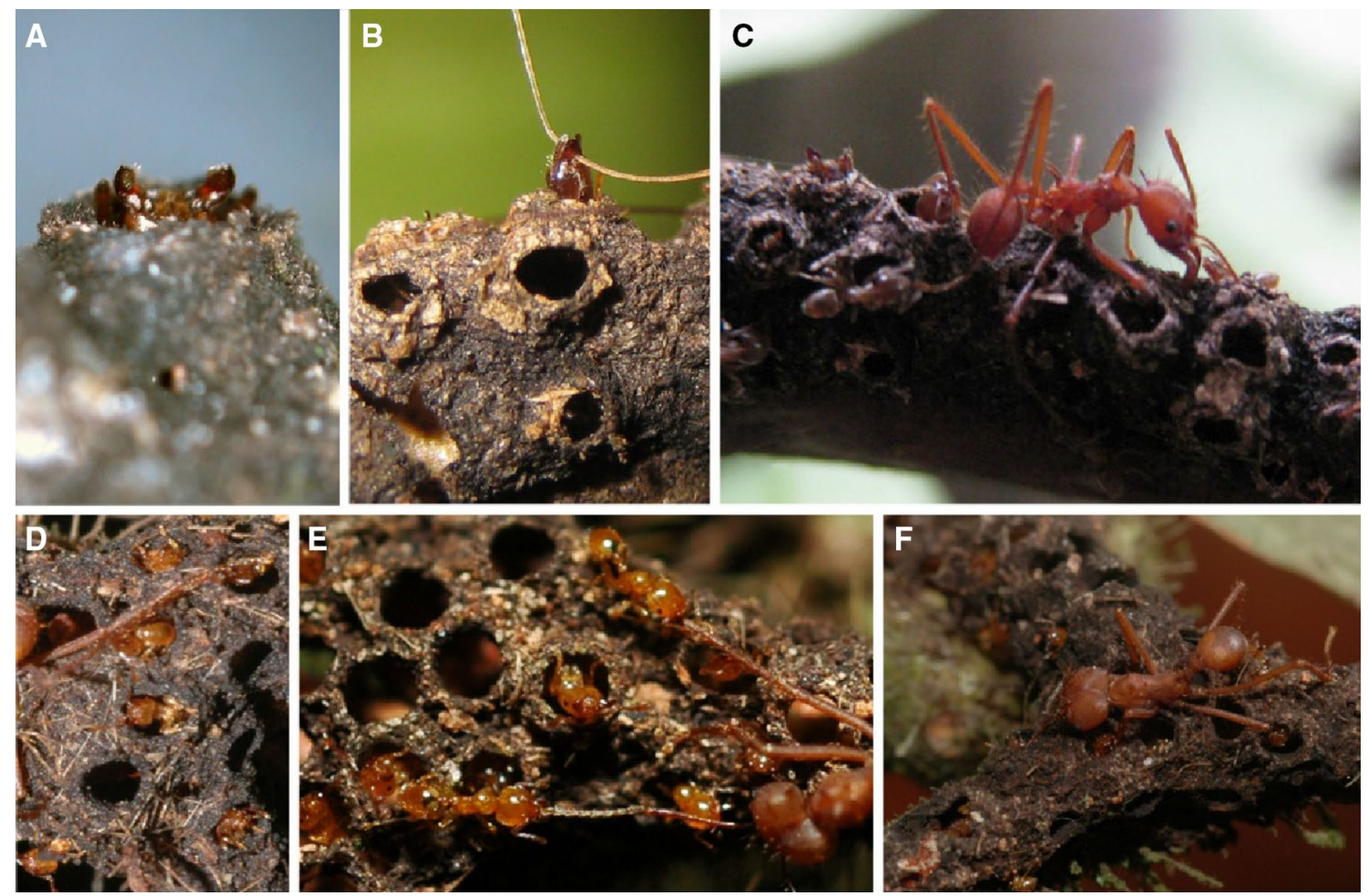

Fig. 2 Function of Az. brevis and Al. decemarticulatus galleries. If the Az. brevis colony is disturbed, the workers take up positions beneath the holes and keep their mandibles wide open (a), grasping any item possible (b). In the latter photo, it is a strand of the first author's hair, but usually Az. brevis ants grasp the legs or anten- nae of other arthropods (c). The prey is immobilised and killed. Al. decemarticulatus workers guard the holes throughout the day (d), and the appendices of the prey walking on the galleries are immediately caught and the prey immobilised $(\mathbf{e}, \mathbf{f})$ 
Fig. 3 Experiment to test whether the galleries serve as traps for Az. brevis and $\mathrm{Al}$. decemarticulatus colonies. Atta workers were placed on inhabited branches of the respective host tree. The Atta ants were then monitored for $30 \mathrm{~min}$ and classified into three categories: remained free- $\mathrm{F}$, captured-C or escaped-E. Significant differences between Az. brevis and Al. decemarticulatus were analysed with a non-parametric Mann-Whitney $U$ test. Further details are in the text. $* * P \leq 0.005, * P \leq 0.05, n s$ not significant
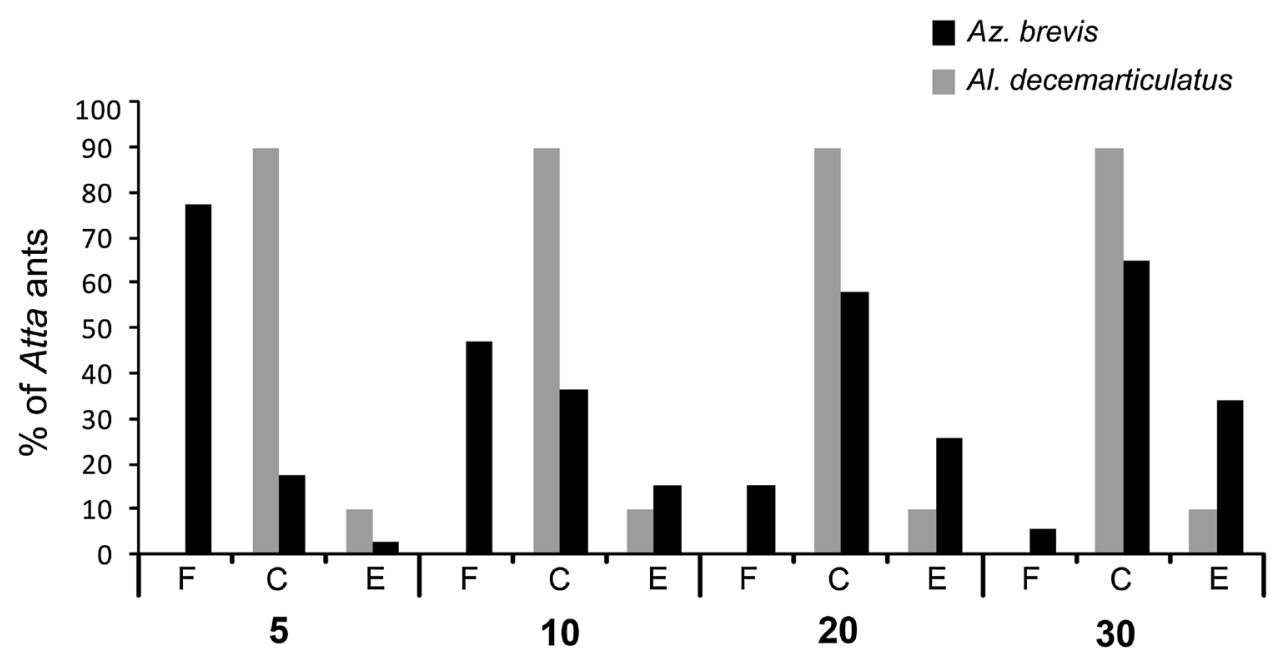

Minutes of the Atta individuals avoided capture and dropped off the tree (Fig. 3).

In contrast, $90.1 \%$ of the 30 Atta workers that were placed on the branches of Al. decemarticulatus-occupied trees were captured within the first 5 min (Figs. 2d-f, 3). Al. decemarticulatus captured the At. cephalotes workers significantly quicker than did the Az. brevis workers (after 5 min: Mann-Whitney $U, z=-5.383, p=0.000, n=57$ ), and even after $20 \mathrm{~min}$, the cumulated capture rate was significantly lower for Az. brevis than for Al. decemarticulatus (Mann-Whitney $U, z=-2.668, p=0.008, n=57$ ). After $30 \mathrm{~min}$, the capture rate was not significantly different (Mann-Whitney $U, z=-1.777, p=0.076, n=57$ ), although in the Allomerus experiments, more Atta individuals were caught (Fig. 3).

The behaviour, which led to captures by both Az. brevis and Al. decemarticulatus, was similar. If the Atta ants inserted one of their legs into one of the gallery's holes, the individual beneath the hole immediately grasped that leg with its mandibles (Fig. 2). Other workers rapidly approached and grasped the other legs and antennae of the prey. Once immobilised, the insect was killed. The Azteca workers very often simply discarded the dead insects, rarely they cut them into pieces and transported parts into the domatia; the rest remained on the carton gallery (Fig. S2, supplementary material). On the contrary, the Allomerus workers always transported the prey to the nearest domatium.

\section{Discussion}

Altogether, our results show a structural and functional convergence in the galleries built by these two ant species that belong to different subfamilies. The galleries result from a similar construction process and the slight variations observed in terms of gallery diameter and hole width may be correlated to the size of the workers (supplementary Table 1). The gallery walls of both ant species are strengthened with fungal mycelia of the order Chaetothyriales (Nepel et al. 2014), a group of Ascomycetes which are frequently found in associations between ants and plants (Voglmayr et al. 2011; Vasse et al. 2017). The structural convergence of the galleries is accompanied by a convergence in their function, as both ant species use them to capture and kill other arthropods. Az. brevis and Al. decemarticulatus workers hide behind the holes and grasp appendices such as the antennae or legs of insects that settle on the gallery, collectively immobilize them, and kill them. Thanks to this ambushing strategy, both ant species are able to capture arthropods whose bodies are much bigger than their own such as the Atta workers in our experiment or long-horned grasshoppers and syrphid flies the remains of which are frequently found on the galleries of Az. brevis (Fig. S2, supplementary material).

A closer look at the two systems reveals differences. First, the material used in construction differs considerably. While Al. decemarticulatus uses plant trichomes as its primary building material and particles of tissue from the inner domatia walls to paste the trichomes together and to favour the growth of the associated fungus (Dejean et al. 2005; Ruiz-González et al. 2011), Az. brevis exclusively uses excavated pith, pieces of bark, or epiphyll leaflets. The use of plant trichomes by Al. decemarticulatus might be attributed to its high specificity with its densely hirsute host plant, H. physophora (Grangier et al. 2009). Az. brevis is more generalist and also found elsewhere than on T. macrophyllum including on Licania sp. (Chrysobalanaceae), Lonchocarpus sp. (Fabaceae), Grias sp. (Lecythidaceae), Myriocarpa sp. (Urticaceae), and Ocotea nicaraguensis 
(Lauraceae) (Longino 2007b; Nepel et al. 2014), none of which bear trichomes as does Hirtella. Such a difference in the construction material might partly explain the greater instability of the Az. brevis galleries, which were often damaged when the prey struggled, whereas those constructed by Al. decemarticulatus always remained intact.

Second, there are behavioural divergences. The first difference could be observed at the beginning of the predation sequence. Az. brevis workers did not seem to wait for prey, and only appeared beneath the holes after the branches were disturbed or after an alarm was triggered, whereas $A l$. decemarticulatus constantly guarded them (pers. observation). Moreover, compared to the congeneric species $A l$. octoarticulatus, Al. decemarticulatus invests more time and energy in gallery construction and use to the detriment of leaf patrolling (Orivel et al. 2017). This may explain why, in the experiment with Atta workers, Al. decemarticulatus caught the Atta workers much quicker and more often than did Az. brevis. Furthermore, Az. brevis was regularly observed to simply discard the dead insects from the branches. This behaviour did not only concern the introduced Atta workers but was also observed for other potential prey insects (e.g., mosquitoes, treehoppers, and small lepidopteran larvae) and contrasts with the behaviour of $A l$. decemarticulatus. Unlike Az. brevis, which tends "herds" of coccids of the genus Cryptostigma (Fig. S1C, supplementary material) that supply the ants not only with carbohydrate-rich exudates, but may also provide protein and lipids if eaten (Gullan 1997; Billick et al. 2007); Al. decemarticulatus does not tend coccids and is mainly a predatory species. This difference in the diet of the two ant species might explain the observed variation in prey capture and indicate a different level of specialization in their hunting strategies and thus different functions of the galleries.

Indeed, Al. decemarticulatus use the galleries mainly for prey capture. In contrast, Az. brevis galleries seem to have mainly a protective function. As the main nest is spread over the entire tree and located in branches bearing a cavity of only a few millimetres in diameter (see Supplementary Fig. S2 B, C), traffic inside this elongated nest (estimated up to 100-m-long if all of the branches are placed end-toend) may be difficult. The runway galleries allow Az. brevis to move quickly and safely without being exposed to enemies and in case of danger to defend the nest efficiently from predators. The use of killed enemies as a food source seems to be a secondary benefit rather than the main function of the Az. brevis galleries.

This dual function, prey capture, and defense, emerged from the structure of the galleries, as the holes allow the workers to enter and exit the galleries elsewhere other than just at the extremities. Since both studied ant species are associated with ant-plants, the defensive/predatory roles of the galleries also benefit the plant in terms of biotic protection against herbivores as shown in the experiment with leaf-cutter ants (but see also Orivel et al. 2017).

Acknowledgements Open access funding provided by University of Vienna. We are grateful to Jack Longino for identifying the Az. brevis, to Markus Schmidt and Alexander Kainz for carrying out part of the experiments with Atta and Az. brevis, to Céline Leroy for her help in the field, and to Andrea Yockey-Dejean for editing the manuscript. We would like to thank the Laboratoire Environnement de Petit Saut and the Nouragues Research Station in French Guiana and the staff of the Estación Tropical La Gamba in Costa Rica for furnishing logistical assistance. Financial support was provided by a fellowship from the Fondation pour la Recherche sur la Biodiversité (Research Agreement No. AAP-IN-2009-050). All fieldwork and sampling in Costa Rica were conducted with the permission of SINAC (Sistema Nacional de Areas de Conservación de Costa Rica of the Ministry of Environment and Energy-MINAE) to V.E.M. (No. 183-2010-SINAC, INU-AWSA-001-16). JL's financial support was provided by a Ph.D. fellowship from the Fond Social Européen (FSE) and by a Nouragues research grant from the Centre National de la Recherche Scientifique (CNRS). This study has benefited from an "Investissement d'Avenir" grant managed by the Agence Nationale de la Recherche (CEBA, ref. ANR-10-LABX-0025).

Open Access This article is distributed under the terms of the Creative Commons Attribution 4.0 International License (http:// creativecommons.org/licenses/by/4.0/), which permits unrestricted use, distribution, and reproduction in any medium, provided you give appropriate credit to the original author(s) and the source, provide a link to the Creative Commons license, and indicate if changes were made.

\section{References}

Anderson C, McShea DW (2001) Intermediate-level parts in insect societies: adaptive structures that ants build away from the nest. Insect Soc 48:291-301

Billick I, Hammer S, Reithel JS, Abbot P (2007) Ant-aphid interactions: are ants friends, enemies, or both? Ann Entomol Soc Am 100:887-892. doi:10.1603/0013-8746(2007)100[887:AIAAFE]2 .0.CO

Dejean A, Quilichini A, Delabie JHC, Orivel J, Corbara B, Gibernau M (2004) Influence of its associated ant species on the life history of the myrmecophyte Cordia nodosa in French Guiana. J Trop Ecol 20:701-704. doi:10.1017/S026646740400183X

Dejean A, Solano PJ, Ayroles J, Corbara B, Orivel J (2005) Insect behaviour: arboreal ants build traps to capture prey. Nature 434:973. doi:10.1038/434973a

Gullan P (1997) Relationships with ants. In: Ben-Dov Y, Hodgson CJ (eds) Soft scale insects-their biology, natural enemies and control. Elsevier, Amsterdam, pp 351-373

Grangier J, Dejean A, Malé PJG, Solano PJ, Orivel J (2009) Mechanisms driving the specificity of a myrmecophyte-ant association. Biol J Linnn Soc 97:90-97. doi:10.1111/j.1095-8312.2008.01188.x

Janzen DH (1983) Costa Rican natural history. Univ. of Chicago Press, Chicago

Leroy C, Jauneau A, Quilichini A, Dejean A, Orivel J (2008) Comparison between the anatomical and morphological structure of leaf blades and foliar domatia in the ant-plant Hirtella physophora (Chrysobalanaceae). Ann Bot 101:501-507. doi:10.1093/aob/ mcm 323 
Longino JT (2007a) A taxonomic review of the genus Azteca in Costa Rica and a global revision of the aurita group. Zootaxa 1491:1-63

Longino JT (2007b) The ants of Costa Rica. http://academic.evergreen.edu/projects/ants/genera/azteca/species/brevis/brevis.html. Accessed 21 Sept 2016

Mayer VE, Voglmayr H (2009) Mycelial carton galleries of Azteca brevis (Formicidae) as a multi-species network. Proc R Soc B 276:3265-3273. doi:10.1098/rspb.2009.0768

Nepel M, Voglmayr H, Schönenberger J, Mayer VE (2014) High diversity and low specificity of chaetothyrialean fungi in carton galleries in a Neotropical ant-plant association. PLoS One 9(11):e112756. doi:10.1371/journal.pone.0112756

Orivel J, Lambs L, Malé PJG, Leroy C, Grangier J, Otto T, Quilichini A, Dejean A (2011) Dynamics of the association between a longlived understory myrmecophyte and its specific associated ants. Oecologia 165:369-376. doi:10.1007/s00442-010-1739-5

Orivel J, Malé PJG, Lauth J, Roux O, Petitclerc F, Dejean A, Leroy C (2017) Trade-offs in an ant-plant-fungus mutualism. Proc R Soc B 284:20161679

Ruiz-González MX, Malé PJG, Leroy C, Dejean A, Gryta H, Jargeat P, Quilichini A, Orivel J (2011) Specific, non-nutritional association between an ascomycete fungus and Allomerus plantants. Biol Lett 7:475-479. doi:10.1098/rsbl.2010.0920

Schmidt MR (2001) Interactions between Tetrathylacium macrophyllum (Flacourtiaceae) and its live-stem inhabiting ants, M.Sc. thesis. University of Vienna, Vienna

Solano PJ, Durou S, Corbara B, Quilichini A, Cerdan P, BelinDepoux M, Delabie JHC, Dejean A (2003) Myrmecophytes of the understory of French Guianian rainforests: their distribution and their associated ants. Sociobiology 41:605-614

Tennant LE (1989) A new ant-plant, Tetrathylacium costaricense. Symp Ants Plants Oxford, p 27

Vasse M, Voglmayr H, Mayer VE, Gueidan C, Nepel M, Moreno L, de Hoog S, Selosse M-A, McKey D, Blatrix R (2017) A fungal phylogenetic perspective of the association between ants (Hymenoptera: Formicidae) and black yeasts (Ascomycota: Chaetothyriales). Proc R Soc B (in press)

Voglmayr H, Mayer VE, Maschwitz U, Moog J, Djieto-Lordon C, Blatrix R (2011) The diversity of ant-associated black yeasts: insights into a newly discovered world of symbiotic interactions. Funct Biol 115:1077-1091. doi:10.1016/j.funbio.2010.11.006 